\title{
Uso de la evaluación en 360 grados para medir competencias en residentes de programas de postítulo de especialidades médicas: Revisión de la literatura
}

\author{
Use of the 360-degrees assessment tool to assess competencies in residents of postgraduate \\ programs of medical specialties: A literature review \\ Diego Correa Fernández', Claudia González Gallardo
}

\section{Resumen:}

En distintos países, han surgido modelos educacionales que definen el marco de competencias necesarias para que todo médico especialista pueda dar respuesta a los constantes cambios que está viviendo la sociedad. Para estos modelos es fundamental que cada rol y competencia sea evaluado correctamente para garantizar su óptima adquisición. Uno de los instrumentos propuestos en la literatura internacional para realizar esto es la evaluación en $360^{\circ}$, que además entrega una retroalimentación oportuna al residente. El objetivo de este trabajo es realizar un artículo de revisión de la literatura sobre la evaluación en $360^{\circ}$.

La evaluación en $360^{\circ}$ es una de las nuevas herramientas que permite dar una evaluación global de las competencias médicas en residentes de programas de especialidades a nivel de posgrado. Consiste en una pauta de observación estructurada que entrega al residente una retroalimentación desde distintos puntos de vista de su práctica cotidiana, al incluir la información obtenida de diferentes grupos de interés para el residente.

Este artículo incluye aspectos relacionados con los componentes y racionalidad educacional del instrumento de evaluación, proceso de elección de los evaluadores incluyendo médicos, profesionales de la salud, personal administrativo, pacientes y familiares. Adicionalmente se analizan aspectos del diseño y aplicación de la evaluación en $360^{\circ}$ o retroalimentación de múltiples fuentes.

\begin{abstract}
:
In different countries, educational models have emerged, definning defining a competence framework needed for every medical specialist to respond to the constant changes that society is experiencing. For these models, it is essential that each role and competence be evaluated correctly to guarantee their optimal acquisition. One of the instruments proposed in the international literature to do this is the 360-degree assessment, which also provides timely feedback to the resident.

The 360-degree assessment is one of the new tools that allows giving a global evaluation of the medical competences in residents of specialty programs at the postgraduate level. It consists of a structured observation guide that gives the resident feedback from different points of view of their daily practice, by including the information obtained from different groups of interest for the resident. This article includes aspects related to the components and educational rationale of this assessment instrument, the selection process of examiners including medical doctors, health professions, and administrative personnel, patients and their relatives additionally, it analyzed aspects related the design and administration of the 360-degree assessment or multisource feedback.
\end{abstract}

Palabras clave: competencia; evaluación en 360 grados; retroalimentación de múltiples fuentes; residentes; posgrado.

Keywords: competence; 360-degree assessment; multisource feedback; residents; postgraduate.

Fecha de envío: 15 de febrero de 2019 - Fecha de aceptación:25 de marzo de 2020

\section{Introducción:}

La práctica médica está viviendo una gran transformación gracias, entre otras, al impacto que han tenido las fuerzas sociales en la medicina (Morán-Barrios \& De Gauna-Bahillo, 2010). Además, la sociedad está cada vez más exigente y se genera una gran brecha entre lo que esperan los pacientes de la práctica médica y lo que

(1) Departamento Otorrinolaringología, Pontificia Universidad Católica de Chile

Autor de correspondencia:dpcorrea3009@gmail.com 
en realidad los servicios están entregando (Neufeld et al., 1998; Carroll, 1999). Por ello, es necesario que el profesional de salud no sólo tenga buenas habilidades clínicas, sino que también cuente con otras competencias que le permitan adaptarse a los nuevos tiempos. Con este panorama surge la pregunta: ¿Cómo puede la educación médica responder a estas demandas para formar mejor a sus médicos? Frente a esta realidad, distintas instituciones han propuesto modelos de competencias que todos los médicos especialistas deben adquirir. Entre ellos destaca como referentes el modelo CanMEDS del Royal College of Physicians and Surgeons en Canadá (Frank, 2005) para la educación de postgrado en especialidades médicas.

Este modelo propone organizar la formación de los residentes en torno a 7 roles: el médico experto, comunicador, colaborador, líder, profesional, académico y promotor de salud; junto con esto se define un conjunto de competencias para cada uno de ellos. Durante los últimos años, este modelo ha sido el que más ha ganado popularidad en todo el mundo incorporándose en la formación de residentes en Reino Unido, Países Bajos, Australia y Nueva Zelanda (Chou et al., 2008). En Chile este proceso no ha sido la excepción, y desde el 2010 la Facultad de Medicina de la Pontificia Universidad Católica de Chile (PUC) ha trabajado en conjunto con el Royal College of Physicians and Surgeons of Canada para implementar el modelo CanMEDS en la formación de los residentes de programas de postítulo de especialidades médicas de la Universidad(“Escuela de Medicina UC la primera institución a nivel internacional en ser acreditada por el Royal College of Physicians and Surgeons of Canada - Escuela de Medicina," 2016).

Para lograr esta implementación es necesario que los distintos programas de especialidades médicas tomen como marco referencial a CanMEDS, incorporando estas competencias en su currículo. Además, es indispensable que exista un sistema de evaluación sólido y coherente con cada uno de los roles y competencias. Esto se debe a que la evaluación es fundamental dentro del proceso de aprendizaje, porque los estudiantes pueden mejorar su desempeño si la utilizan como parte integral de su aprendizaje (Ministerio de Educación, 2006; Schuwirth \& Van der Vleuten, 2011). Otro objetivo de la evaluación es corroborar la adecuada adquisición de cada una de las competencias declaradas en los programas (Nolla-Domenjó, 2009).

Realizar una evaluación adecuada no es fácil, ya que el docente se ve enfrentado a un residente con distintos atributos que se desempeña en distintos contextos de su práctica clínica; además no sabe cuáles de estos podrá evaluar de forma confiable, ni menos como hacer que esto impacte en la educación de ellos (Epstein, 2007). Para solucionar estas interrogantes, han surgido nuevas herramientas de evaluación para los residentes, dentro de ellas la evaluación basada en el lugar de trabajo (Norcini \& Burch, 2007). Este sistema de evaluación se refiere a evaluar lo que los residentes hacen realmente en la práctica diaria ( Miller \& Archer, 2010). Dentro de sus ventajas está la posibilidad de entregar retroalimentación creíble al residente sobre su desempeño, de forma oportuna y enfocado en el contexto clínico en que ocurre (Miller, 1990; Norcini \& Burch, 2007). Esta característica le entrega un potencial formativo a este tipo de evaluación, ya que permite que el residente mejore en su práctica diaria y dirija su aprendizaje hacia los resultados deseados ( Norcini \& Burch, 2007; Miller \& Archer, 2010). Tal es la fuerza de la retroalimentación, que estudios recientes plantean que si esta es sistemática, oportuna y entregada por una fuente creíble, podría modificar el desempeño clínico de los estudiantes, sin embargo aún no hay claridad al respecto (Miller \& Archer, 2010).

De acuerdo a la literatura, existen algunos roles CanMEDS más evaluados que otros, como por ejemplo el experto médico, cuyo énfasis son los conocimientos y destrezas clínicas del residente (Puddester et al., 2015), dejando de lado el resto de los roles, llamados intrínsecos. La situación en los programas de especialidad en la PUC no difiere con la realidad planteada en Canadá. Por lo anterior, el esfuerzo debe enfocarse en evaluar de mejor manera estos roles intrínsecos, distintos al médico experto (Chou et al., 2008; Puddester et al., 2015). Dado que el aprendizaje de los roles intrínsecos se produce principalmente en el lugar de trabajo con el resto del equipo, se debe buscar instrumentos de evaluación que midan este tipo de roles en el lugar de trabajo y que involucren la visión de otros miembros del equipo de salud (Whitehead et al., 2011).

Uno de los instrumentos propuestos en la literatura que cumple con esto es la evaluación en $360^{\circ}$, pauta estructurada que entrega una mirada del desempeño médico desde distintos puntos de vista, haciendo énfasis en los roles intrínsecos (Wood et al., 2006).

Por lo anteriormente planteado, el propósito de este trabajo es realizar un artículo de revisión sobre la evaluación en $360^{\circ}$ para posteriormente utilizarlo como base para la creación de un instrumento nuevo.

\section{Evaluación en $360^{\circ}$}

La evaluación en $360^{\circ}$ o retroalimentación de múltiples fuentes o MSF, por sus siglas en inglés (multiple source feedback) consiste en una pauta de observación estructurada que entrega al residente una retroalimentación desde distintos puntos de vista de su práctica cotidiana, al incluir la información obtenida de diferentes grupos de interés para el residente: profesionales médicos, profesionales no médicos (enfermeras, tecnólogos médicos, nutricionistas, kinesiólogos, etc.), técnicos paramédicos, administrativos, pacientes, etc. 
(Ao et al., 2014). Este instrumento se sustenta en la premisa que la retroalimentación de rutina entregado por una fuente creíble mejora el desempeño de los médicos(Chou et al., 2008).

En su origen, este instrumento fue una herramienta de evaluación utilizado en la industria. Tal ha sido su impacto en el desarrollo profesional, que las principales empresas de EE.UU lo usan de forma rutinaria y cerca del $90 \%$ de los gerentes lo encuentra útil (Wood et al., 2006). Sin embargo, en el ámbito de la educación médica, los resultados son variados y poco concluyentes. Uno de los puntos demostrado es el alto valor educativo que perciben los docentes de la evaluación en $360^{\circ}$.

\section{Elección de los evaluadores}

Este instrumento de evaluación consiste en que distintos miembros del equipo de trabajo actúan como evaluadores de un individuo (Bunk, 1994; Martínez et al., 2008; Berger et al., 2012; Dwyer et al., 2014). En el ámbito clínico, se sugiere que los evaluadores sean personas que compartan día a día con el residente, para que logren tener un conocimiento directo del comportamiento de este (Bandiera \& Sherbino, 2006; Wood et al., 2006); así podrán proporcionar una retroalimentación apropiada sobre su desempeño (Wood et al., 2006). Dentro de los evaluadores están a los profesionales médicos, como los médicos staffs, residentes pares, o estudiantes de pregrado de medicina; profesionales no médicos, como las enfermeras, psicólogos y farmacéuticos; técnicos paramédicos, administrativos; e incluso los pacientes y sus familias. Además, siempre es recomendado que el residente evaluado realice una autoevaluación (Bandiera \& Sherbino, 2006; Wood et al., 2006).

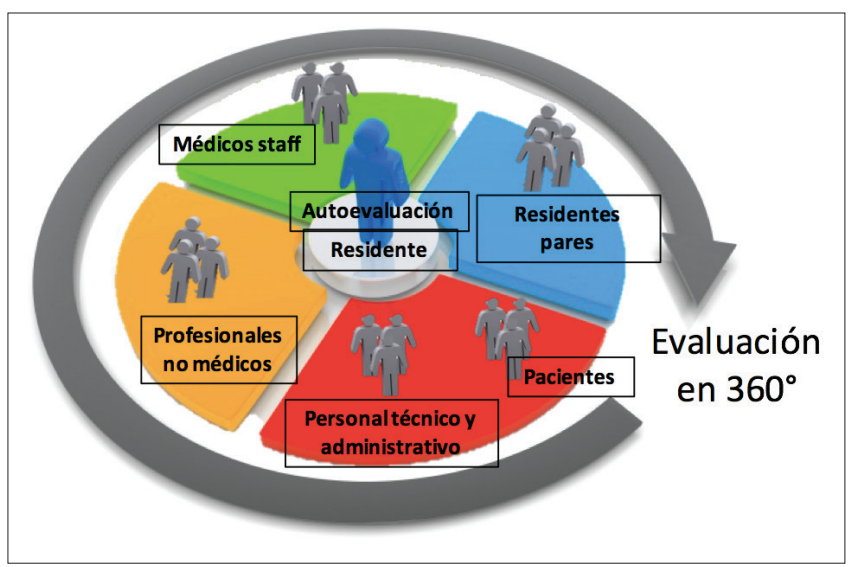

Figura 1: Esquema de la evaluación en $360^{\circ}$ de los residentes.
En relación a como se realiza la elección de los evaluadores, la literatura no ha llegado a un consenso: algunos han demostrado que el evaluado podría nombrar a su propio evaluador, sin sesgos en el resultado (Wood et al., 2006; Allerup et al., 2007; Berk, 2009) mientras que otros prefieren no dejar la elección en manos del evaluado, porque esta disminuye la validez de los resultados (Wood et al., 2006).

Por otro lado, hay que establecer la cantidad de evaluadores necesarios para disminuir al mínimo todo riesgo de sesgo y lograr aumentar la confiabilidad del instrumento (Bandiera \& Sherbino, 2006). En este sentido, Wood plantea que deben ser entre 6 y 10 evaluadores por individuo, por ejemplo, 5 miembros del equipo de salud no médicos, 3 médicos más la autoevaluación (Berk, 2009). En el caso de incluir pacientes y sus familias, deberían ser más de 50 evaluadores (Wood et al., 2006).

\section{Diseño}

Estas evaluaciones se realizan a través de cuestionarios estructurados, diseñados para evaluar conductas clínicas que se pueden observar y remediar (Bandiera \& Sherbino, 2006). Estas conductas medidas son muy variadas en la literatura, no obstante, siempre el énfasis está en competencias intrínsecas como las habilidades comunicacionales, de trabajo interprofesional y de profesionalismo (Berk, 2009). Los cuestionarios pueden ser iguales o diferentes para cada grupo de distintos evaluadores (Bandiera \& Sherbino, 2006).

En cuanto al número de ítems que debe tener la encuesta, algunos plantean que debieran ser entre 10 a 40 (Bandiera \& Sherbino, 2006). Sin embargo, una gran cantidad de ítems puede aumentar la inconsistencia de la evaluación y no aumentaría la discriminación; por ello se debe proponer el menor número de ítems posibles (Wood et al., 2006).

La escala que se usará en el cuestionario es otro aspecto relevante. De acuerdo a la evidencia disponible, se aconseja dar entre 3 a 7 opciones de respuesta y que estas estén acorde al propósito de la evaluación, además de dejar espacio para comentarios (Wood et al., 2006; Berk, 2009).

La figura 2 muestra un ejemplo de esta pauta. 


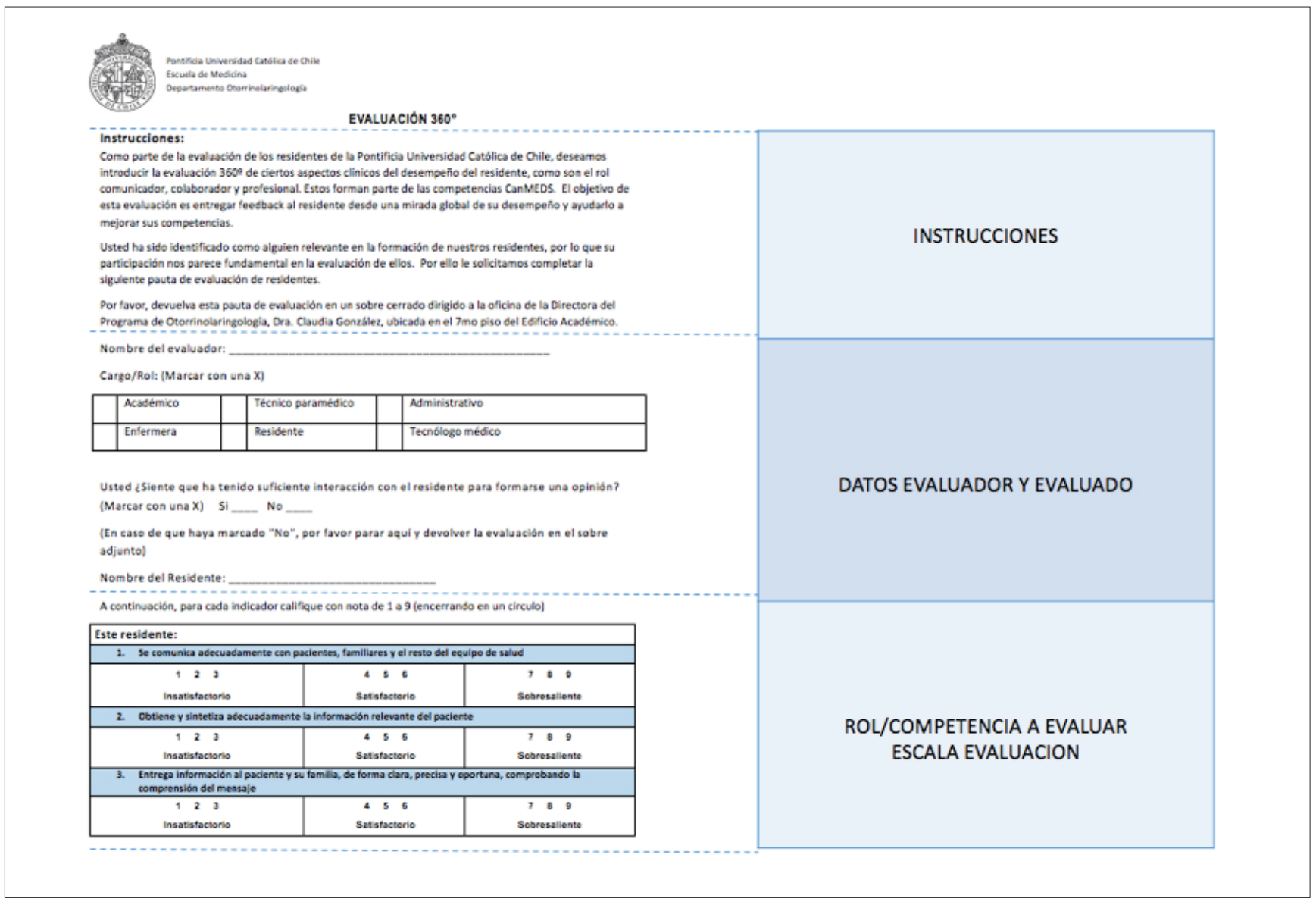

Figura 2: Ejemplo de una pauta de evaluación en $360^{\circ}$.

\section{Aplicación de la evaluación en $360^{\circ}$}

Una vez diseñado el instrumento, se debe aplicar la encuesta. Para lo cual existen 2 opciones: vía online o en papel. Cada una de estas dos modalidades presenta ventajas y desventajas. Para muchos es preferible enviarla de forma online para proteger el anonimato del evaluador y conseguir mayor cantidad y calidad en los comentarios (Berk, 2009); otros, prefieren en papel, ya que los sistemas electrónicos pueden alterar los datos (Wood et al., 2006).

La siguiente etapa es recolectar la información, analizarla y preparar la retroalimentación individual para el evaluado. Esta devolución se realiza en una reunión formal, cara a cara, dirigida por el jefe de cada programa (Wood et al., 2006). En esta reunión, se debe presentar la información recopilada de manera gráfica, reflejando las calificaciones promedios obtenidas por el residente para cada ítem, junto con la calificación promedio obtenida por todos los residentes del nivel para ese mismo ítem (Norcini \& Burch, 2007). También, se debe graficar la autoevaluación que el individuo realizó para cada ítem (Wood et al., 2006). En la Figura 3 se muestra un ejemplo de la esquematización de los resultados para cada residente. Todos los comentarios se incluyen textualmente, pero permanecen anónimos (Norcini \& Burch, 2007). De esta manera, en conjunto, identificarán las áreas donde el individuo se destaca y las que debe mejorar, para así fomentar la reflexión, desarrollar un plan de mejora y lograr beneficiar su desarrollo profesional (Lockyer, 2003; Bandiera \& Sherbino, 2006). Para que esta retroalimentación sea adecuado y logre su propósito, es fundamental preparar y entrenar a las personas que lo realicen (Wood, Hassell et al., 2006).

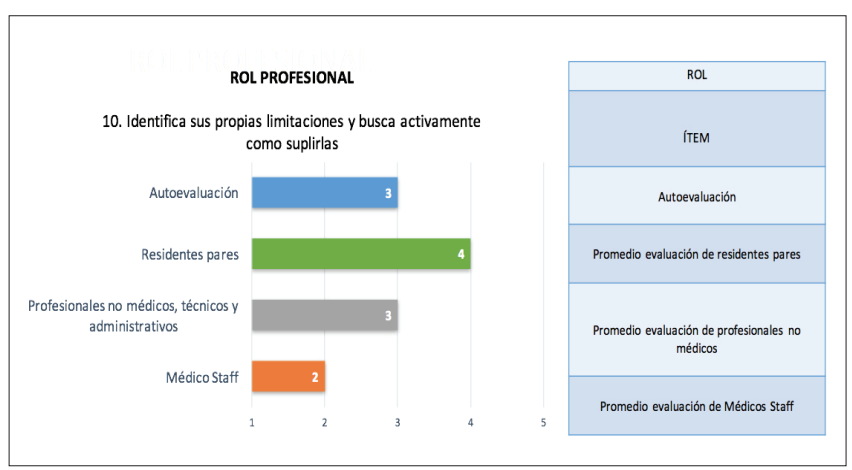

Figura 3: Ejemplo de la esquematización de los resultados de la evaluación en $360^{\circ}$. 


\section{Discusión}

La evaluación en $360^{\circ}$ destaca como una de las nuevas herramientas que permite dar una evaluación global de las competencias de los residentes de especialidades médicas. Si bien, se pueden evaluar distintos tipos de habilidades, el énfasis está en las habilidades comunicacionales, de trabajo interprofesional y de profesionalismo (Berk, 2009). Esto permite que se enfatice su importancia y necesidad para una práctica médica óptima.

Con este instrumento se busca que el residente sea evaluado desde la mirada de la mayor parte del equipo de salud, tanto los profesionales médicos como profesionales no médicos, técnicos y administrativos.

Las principales ventajas de este instrumento son: puede complementar otras evaluaciones, permite hacer visibles valores y comportamientos que son difíciles de evaluar por los métodos tradicionales, se puede adaptar a entornos cambiantes y podría estimular cambios y mejoras en el desempeño de los residentes (Bandiera \& Sherbino, 2006; Wood, et al., 2006).

Por otro lado, también existen algunas desventajas que hay que tener en cuenta cuando se intenta implementar este tipo de instrumentos. Algunas de ellas serían: primero, requiere tiempo para el diseño de los cuestionarios, ya que se requiere que estén muy bien planteados para lograr una herramienta válida que evalúe adecuadamente el comportamiento; segundo, requiere una infraestructura estable para la distribución de la encuesta y contribución continua de los evaluadores; tercero, requiere un número apropiado de evaluadores para tener una buena confiabilidad; finalmente, puede haber cierta reticencia, por parte de los residentes, de ser evaluados por miembros del equipo de salud que no sean médicos (Bandiera \& Sherbino, 2006).

En conclusión, la evaluación en $360^{\circ}$ es un instrumento que permite evaluar el desempeño del residente en el lugar de trabajo, desde una mirada global, al incluir la información obtenida de distintos grupos de interés para el residente. Otra característica fundamental de este tipo de instrumentos es la retroalimentación que proporciona a los residentes, la que debe ser individual y oportuna. Esta característica ha sido de gran relevancia, ya que permite con el uso de este instrumento de evaluación se logre fundamentar y hacer tangible el concepto de la evaluación para el aprendizaje.

Se espera utilizar la información recogida en esta revisión para diseñar un instrumento de evaluación en $360^{\circ}$ que permita evaluar competencias intrínsecas en los residentes de programas de especialidades médicas de la PUC. De esta forma, se contribuirá a que los programas de especialidad de la PUC y los especialistas formados en ellos sean reconocidos por poseer estas competencias.

\section{Contribuciones y reconocimientos:}

-Sin fuentes de financiamiento

-Sin conflicto de interés

\section{Referencias:}

Allerup P, Aspegren K, Ejlersen E, Jørgensen G, Malchow-Møller A, Møller MK, Pedersen KK, Rasmussen OB, Rohold A \& Sørensen B. (2007). Use of 360-degree assessment of residents in internal medicine in a Danish setting: a feasibility study. Medical Teacher 29, 166-70.

Ao P, Kahlon S, Daniels V \& Goldstein C. (2014). Development of a multisource feedback tool in the assessment of intrinsic CanMEDS Roles for senior internal medicine residents. Presented: Oral Podium Presentation International Conference on Residency Education, Toronto, Ontario, Canada.

Bandiera G, Sherbino J. \& Frank J. (2006). The CanMEDS assessment tools handbook. guide to assessment methods for the CanMEDS Competencies. The Royal College of Physicians and Surgeons of Canada

Berger E, Chan MK, Kuper A, Albert M, Jenkins D, Harrison M \& Harris I. (2012). The CanMEDS role of Collaborator: How is it taught and assessed according to faculty and residents? Paediatrics and Child Health (Canada) 17, 557-560.

Berk RA. (2009). Using the 360 multisource feedback model to evaluate teaching and professionalism. Medical Teacher 31, 1073-1080.

Bunk G. (1994). in Initial and Continu- ing Vocational Training in the Federal Republic of Germany. Eur Vocat Train J 1, 8-14.

Carroll T. (1999). Multidisciplinary collaboration: a method for measurement. Nursing Administration Quarterly 23, 86-90.

Clares P, Martínez Juárez M. \& Muñoz Cantero JM. (2008). Formación basada en competencias en educación sanitaria: Aproximaciones a enfoques y modelos de competencia. RELIEVE - Revista Electronica de Investigacion Y Evaluacion Educativa 14, 1-23.

Chou S, Cole G, McLaughlin K. \& Lockyer J. (2008). CanMEDS evaluation in Canadian postgraduate training programmes: Tools used and programme director satisfaction. Medical Education 42, 879-886.

Dwyer T, Takahashi SG, Hynes MK, Herold J, Wasserstein D, Nousiainen M. et al. (2014). How to assess communication, professionalism, collaboration and the other intrinsic CanMEDS roles in orthopedic residents: Use of an objective structured clinical examination (OSCE). Canadian Journal of Surgery 57, 230-236. 
Epstein RM. (2007). Medical education - Assessment in medical education. New England Journal of Medicine 356, 387-396.

Escuela de Medicina UC la primera institución a nivel internacional en ser acreditada por el Royal College of Physicians and Surgeons of Canada - Escuela de Medicina. (2016). Accedido en https://medicina. uc.cl/noticias/escuela-de-medicina-uc-la-primera-institucion-a-nivel-internacional-en-ser-acreditada-por-el-royal-college-of-physicians-and-surgeons-of-canada/ el 15 de diciembre de 2018.

Frank JR. (2005). The CanMEDS 2005 Physician Competency Framework. Framework. Otawa: Royal College of Physicians and Surgeons of Canada.

Lockyer J. (2003). Multisource feedback in the assessment of physician competencies. Journal of Continuing Education in the Health Professions 23, 4-12.

Miller A. \& Archer J. (2010). Impact of workplace based assessment on doctors' education and performance: a systematic review. BMJ (Clinical Research Ed.) 341, c5064.

Miller GE. (1990). The assessment of clinical skills/competence/ performance. Academic Medicine 65, S63-7.

Ministerio de Educación. (2006). Evaluación para el aprendizaje: Enfoque y materiales prácticos para lograr que sus estudiantes aprendan más y mejor 307. Accedida en https://bibliotecadigital. mineduc.cl/handle/20.500.12365/2055 el 05 de enero de 2019

Morán-Barrios J. \& De Gauna-Bahillo PR. (2010). ¿Reinventar la formación de médicos especialistas? Principios y retos. Nefrologia 30, 604-612.

Neufeld VR, Maudsley RF, Pickering RJ, Turnbull JM, Weston WW, Brown MG \&Simpson JC.(1998). Educating future physicians for Ontario. Academic 11, 1133-48.
Nolla-Domenjó, M. (2009). La evaluación en educación médica: Principios básicos. Educación Médica 12, 223-229.

Norcini J. \& Burch V. (2007). Workplace-based assessment as an educational tool: AMEE Guide No. 31. Medical Teacher 29, 855-71.

Puddester D, MacDonald CJ, Clements D, Gaffney J, \& Wiesenfeld L. (2015). Designing faculty development to support the evaluation of resident competency in the intrinsic CanMEDS roles: practical outcomes of an assessment of program director needs. BMCMedical Education 15, 100.

Schuwirth LWT. \& Van der Vleuten CPM. (2011). Programmatic assessment: From assessment of learning to assessment for learning. Medical Teacher 33, 478-485.

The Competency-Based Management Blog: Multi-source/360-Degree Feedback (2012) (Part 9 of 10 of HRSG's Guide to Performance Management through Competencies). Accedido en http://blog. competencycore.com/2012/10/multi-source360-degree-feedback-part-9.html el 15 de enero 2019.

Whitehead C, Martin D, Fernandez N, Younker M, Kouz R, Frank J. \& Boucher A. (2011). 15 Integration of CanMEDS Expectations and Outcomes. Members of the FMEC PG Consortium, 1-24.

Wood L, Hassell A, Whitehouse A, Bullock A. \& Wall D. (2006). A literature review of multi-source feedback systems within and without health services, leading to 10 tips for their successful design. Medical Teacher 28, e185-e191.

Wood L, Wall D, Bullock A, Hassell A, Whitehouse A. \& Campbell I. (2006). "Team observation": a six-year study of the development and use of multi-source feedback (360-degree assessment) in obstetrics and gynaecology training in the UK. Medical Teacher 28, e177-e184. 